\title{
A giant and insidious subphrenic biloma formation due to gallbladder perforation mimicking biliary cystic tumor: A case report
}

\author{
GUWEI JI ${ }^{1}$, FEIPENG ZHU ${ }^{2}$, KE WANG ${ }^{1}$, CHENYU JIAO ${ }^{1}$, ZICHENG SHAO ${ }^{1}$ and XIANGCHENG LI ${ }^{1}$ \\ ${ }^{1}$ Liver Transplantation Center; ${ }^{2}$ Department of Radiology, \\ The First Affiliated Hospital of Nanjing Medical University, Nanjing 210029, P.R. China
}

Received June 9, 2016; Accepted September 20, 2016

DOI: $10.3892 / \mathrm{mco} .2016 .1075$

\begin{abstract}
Gallbladder perforation (GBP) represents a rare, but potentially life-threatening, complication of acute cholecystitis. GBP is subdivided into three categories whereas the development of biloma is extremely rare. The present case study reports on a 40 -year-old man with a 10 -year history of calculus cholecystitis, who was referred to The First Affiliated Hospital of Nanjing Medical University (Nanjing, China) for the surgical treatment of an emerging massive hepatic entity with insidious symptoms and normal laboratory tests. A preoperative imaging study demonstrated the collection with internal septations and mural nodules, but no visible communication with the biliary system. Given the suspected biliary cystic tumor, a laparotomy was performed and the lumen was scattered with papillae. An intraoperative frozen section examination illustrated a simple hepatic cyst. Biochemical analysis of the collection and histopathology of the gallbladder and capsule substantiated the diagnosis of biloma formation due to GBP. The purpose of the present case report was to demonstrate how a pinhole-sized perforation with extravasation of unconcentrated bile from the gallbladder may result in insidious clinical presentation and an undetected leak site. According to the clinicopathological characteristics and composition, formation of biloma should be classified as type IV GBP. To differentiate bilomas with intracystic septations
\end{abstract}

Correspondence to: Professor Xiangcheng Li, Liver Transplantation Center, The First Affiliated Hospital of Nanjing Medical University, 300 Guangzhou Road, Nanjing 210029, P.R. China

E-mail:drxcli@njmu.edu.cn

Abbreviations: GBP, gallbladder perforation; BCT, biliary cystic tumor; CT, computed tomography; RUQ, right upper quadrant; MRI, magnetic resonance imaging; MRCP, magnetic resonance cholangiopancreatography; US, ultrasonography; PTBD, percutaneous transhepatic biliary drainage

Key words: biloma, gallbladder perforation, biliary cystic tumor, calculus cholecystitis, insidious symptoms and mural nodules from BCTs is difficult via a preoperative examination, and the definitive diagnosis should be based on a histological examination. Laparotomy with frozen section examination may be the optimal approach in such a case.

\section{Introduction}

Gallbladder perforation (GBP) represents a rare, but potentially life-threatening, complication of acute cholecystitis, with an incidence rate of $0.8-4.8 \%$ and a mortality rate of $9.5-16 \%$ as reported in recent studies (1-4). The proposed mechanism of GBP is gallbladder wall ischemia and necrosis triggered by intense inflammatory reaction, and intracholecystic hypertension caused by calculi impacted in the cystic duct (5). Acute abdominal pain accompanied by fever or nausea and vomiting portends the onset of perforation. However, clinical discrimination from uncomplicated acute cholecystitis is difficult due to overlapping signs and symptoms, leading to a delay in diagnosis and poor results $(3,6)$. Radiological evaluations serve a vital role in early identification and appropriate intervention. Although focal disruption of the wall is the definitive imaging finding of GBP, small perforations may escape detection, and preoperative diagnosis therefore remains challenging (7).

Based on the clinicopathological findings of Niemeier in 1934 (8), GBP is subdivided into three categories: Type I presents an acute status with generalized biliary peritonitis; type II is characterized as a subacute stage with abscess formation and localized peritonitis; and type III is a chronic procedure, with the development of fistula between gallbladder and another viscus. Subacute perforation is the most common type, followed by acute and chronic perforations, whereas the development of biloma is extremely rare $(5,9)$.

In the present study, a case of a giant and asymptomatic subphrenic biloma formation secondary to GBP without visualized defects, and mimicking a biliary cystic tumor, is reported.

\section{Case report}

In December 2015, a 40-year-old man with a 10-year history of calculus cholecystitis was referred to The First Affiliated Hospital of Nanjing Medical University (Nanjing, China) for 
surgical treatment of an emerging massive subphrenic entity without abdominal pain, fever, jaundice, nausea or omitting. The patient had been admitted to the local hospital two months earlier for conservative treatment of an acute attack of chronic cholecystitis. An initial computed tomography (CT) scan was unremarkable, apart from calculus cholecystitis. The patient's medical history was negative for abdominal trauma and surgery.

An abdominal examination revealed a palpable hard mass in the right upper quadrant (RUQ) without tenderness. Abnormal laboratory findings included mild elevation of total and direct bilirubins (1.7 and $0.8 \mathrm{mg} / \mathrm{dl}$, respectively), alkaline phosphatase (199.3 U/l) and $\gamma$-glutamyltransferase (98.6 U/l). Levels of peripheral blood count (white blood cell count, 5.42×10 $/ 1$; neutrophils, 57\%; hemoglobin, $144 \mathrm{~g} / 1$; platelet count, $187 \times 10^{9} / 1$ ), liver enzymes (aspartate transaminase, 31.3 U/1; alanine transaminase, $34.5 \mathrm{U} / \mathrm{l}$ ) and tumor markers (carcinoembryonic antigen, $1.1 \mathrm{ng} / \mathrm{ml}$; $\alpha$-fetoprotein, $4.0 \mathrm{ng} / \mathrm{ml}$; carbohydrate antigen 19-9, $0.7 \mathrm{U} / \mathrm{ml}$ ) were all within normal limits.

A repeat CT scan revealed a well-circumscribed, hepatic cystic lesion, measuring approximately $20.4 \times 15.4 \mathrm{~cm}$, without enhancement (Fig. 1). Magnetic resonance imaging (MRI) revealed a hepatic mass that was revealed by a homogenously iso-intensity signal on a T1-weighted image, with hyper-intensity and also several iso-intensity septations and small mural nodules on a T2-weighted image (Fig. 2A). Magnetic resonance cholangiopancreatography (MRCP) demonstrated the gallstones and subphrenic collection, without any visible communication with the biliary system (Fig. 2B).

A biliary cystic tumor (BCT), particularly cystadenocarcinoma, was highly suspected, based on the intracystic septations and mural nodules that were demonstrated on the MR images. Therefore, a laparotomy was performed, and an intraoperative examination revealed that the greater omentum had adhered to the undersurface of the entity and gallbladder densely (Fig. 3A). Approximately 4,000 ml dark-yellow fluid was aspirated, and the lumen was scattered with papillae (Fig. 3B). Given the suspected BCT and its malignant potential, a frozen section examination was performed at the time of performing the fenestration, which illustrated a simple hepatic cyst. The cyst wall was peeled off from the hepatic capsule and the right diaphragm with ease following cholecystectomy. Histopathology of the gallbladder illustrated an ulcerative episode of a chronic-recurring calculus cholecystitis with focal granulation tissue formation. Microscopically, the wall was made up of fibrous tissue without epithelial lining, which indicated that the fluid collection produced encapsulation by inducing a mild inflammatory response and fibrosis (Fig. 4). The aspiration fluid contained bile (total bilirubin, $8.3 \mathrm{mg} / \mathrm{dl}$ ), and was negative on culture, further substantiating the diagnosis of biloma. The patient had an uneventful postoperative course and was discharged 7 days after the operation. There was no recollection, or any other associated complaint after follow-up for 6 months.

Written informed consent was obtained from the patient for publication of this case report and accompanying images, and the study was approved by the ethics committee of The First Affiliated Hospital of Nanjing Medical University (Nanjing, China).

\section{Discussion}

Biloma was first coined by Gould and Patel (10), but was redefined as intra- or extra-hepatic bile collection outside the biliary tree with well-demarcated capsule in 1983 by Kuligowska et al (11). Iatrogenic biliary injury, particularly cholecystectomy, is the predominant causative factor, followed by blunt abdominal injury $(12,13)$. In certain cases, spontaneous biloma formation may occur, and this is usually associated with bile duct disruption, but rarely with GBP (13-16). Acute perforation of the gallbladder generally presents as generalized peritonitis followed by sepsis syndrome, ascribed to the abrupt release of concentrated bile into the peritoneal cavity. However, in certain instances, a low-grade and chronic bile leakage from the gallbladder becomes encapsulated to form a biloma.

Abdominal discomfort or pain (predominantly in the RUQ), accompanied by fever, is the most common clinical presentation of bilomas. Jaundice, nausea and vomiting are often present as a result of extrinsic compression of the biliary or digestive system, whereas certain patients may be asymptomatic. Laboratory findings consist of leukocytosis with neutrophilia and liver dysfunction, although the values obtained may be non-specific, and a few patients have no abnormalities at the time of diagnosis (12).

Abdominal ultrasonography (US) is usually the initial modality to detect fluid collections, and the underlying pathology for its non-radiation and accessibility. On US images, bilomas present as well-circumscribed, anechoic and encapsulated masses with or without internal debris and septa $(7,12)$. However, a variety of intraperitoneal cystic lesions have overlapping features, including hematoma, hepatic cyst, abscess and pseudocyst. An enhanced CT scan is a sensitive modality to confirm the collection, as well as for the identification of the distribution and adjacent anatomy (17), whereas the MRI technique is superior in delineating the fluid homogeneity and differential diagnosis. Low-signal intensity within the collection on T2-weighted sequences indicates the presence of components. MRCP sequences are of value to depict the course of the biliary system, and the communication between cystic lesions and bile ducts. CT cholangiography and cholescintigraphy are non-invasive and effective means of demonstrating the presence and site of biliary excretion, complementing the MRCP in selected cases and avoiding the requirement for endoscopic retrograde cholangiopancreatography or percutaneous transhepatic cholangiography $(18,19)$. In exceedingly rare cases, however, a pinhole-sized perforation may escape detection on medical imaging, even when intraoperative cholangiography is performed (20).

Multiple imaging modalities and the clinical history are warranted for the diagnosis of biloma, but the diagnosis is confirmed by demonstrating a communication with the biliary system or the biochemical analysis of the fluid collection. Although rare, BCTs should be suspected for a cystic neoplasm of liver, particularly with septum-like structures and suspicious intracystic components, as in our case study (21). However, hematological analysis and tumor markers are non-specific for distinguishing BCTs from other hepatic cystic masses $(21,22)$. Given the suspected BCT, image-guided needle aspiration cytology or biopsy is not implemented, due to high false 


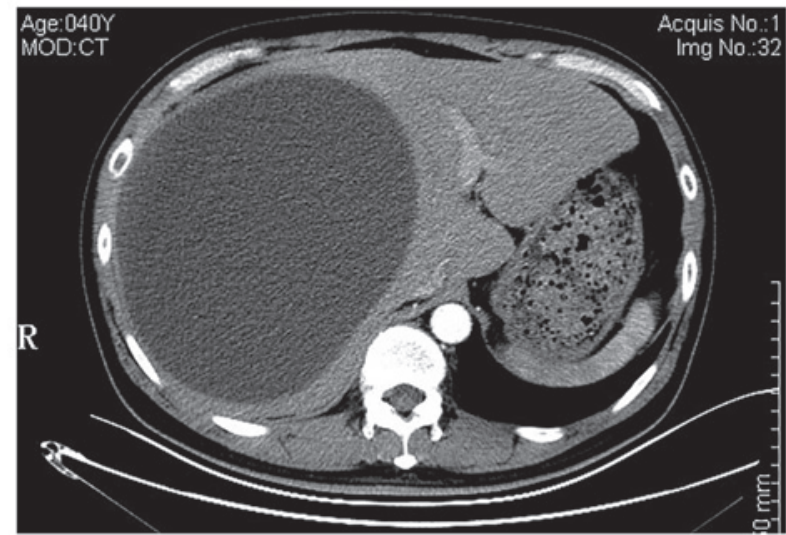

Figure 1. Axial abdominal computed tomography scan, revealing a massive cystic lesion without identifiable capsule, distorting the liver configuration.
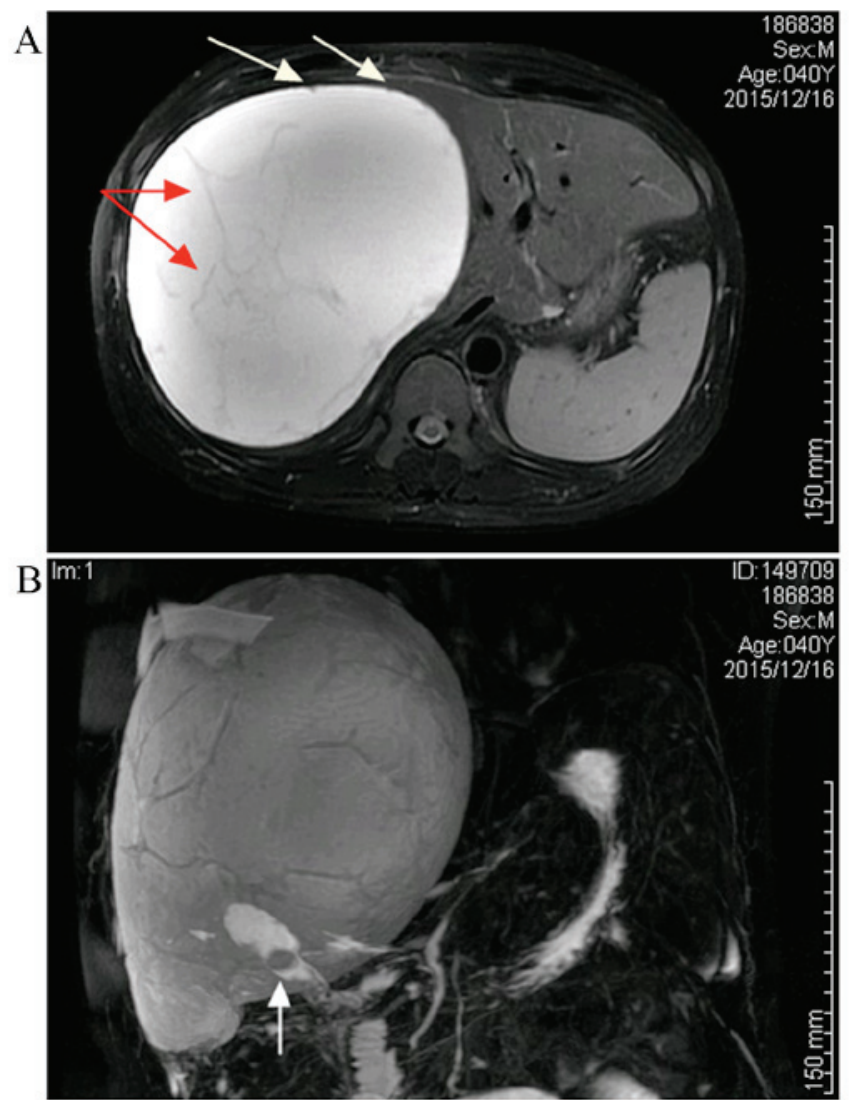

Figure 2. Magnetic resonance imaging and magnetic resonance choangiopancreatography scans of the cystic lesion. (A) The collection was revealed to be hyper-intensive on an axial T2-weighted image, with some iso-intensity septations (illustrated by the red arrows) and small mural nodules (illustrated by the white arrows). (B) A magnetic resonance cholangiopancreatography image illustrating a giant subphrenic collection and gallbladder stones (shown by the white arrow), although there was no visible communication between the lesion and gallbladder.

negative results and the risk of peritoneal dissemination (23). Furthermore, an elevated bilirubin concentration in the aspiration fluid cannot be considered as a conclusive parameter to discriminate bilomas from BCTs with bile duct communication $(24,25)$. In the present case study, intraoperative detection of scattered papillomas highlighted our suspicion of BCT.
A

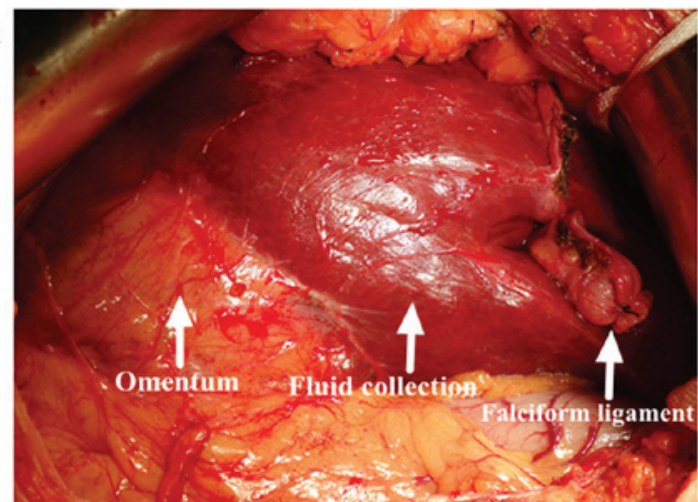

$\mathrm{B}$

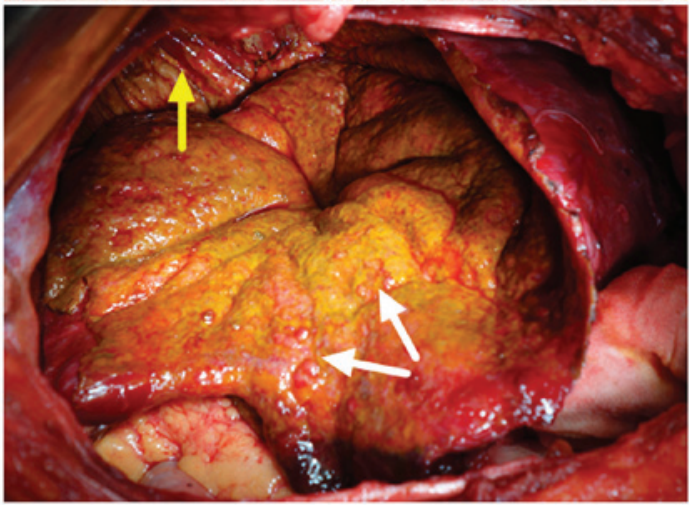

Figure 3. Intraoperative images (A) demonstrating the greater omentum adhered to the undersurface of the collection densely, and (B) scattered papilla inside the capsule (shown by the white arrow) and subphrenic portion of the lesion (shown by the yellow arrow).

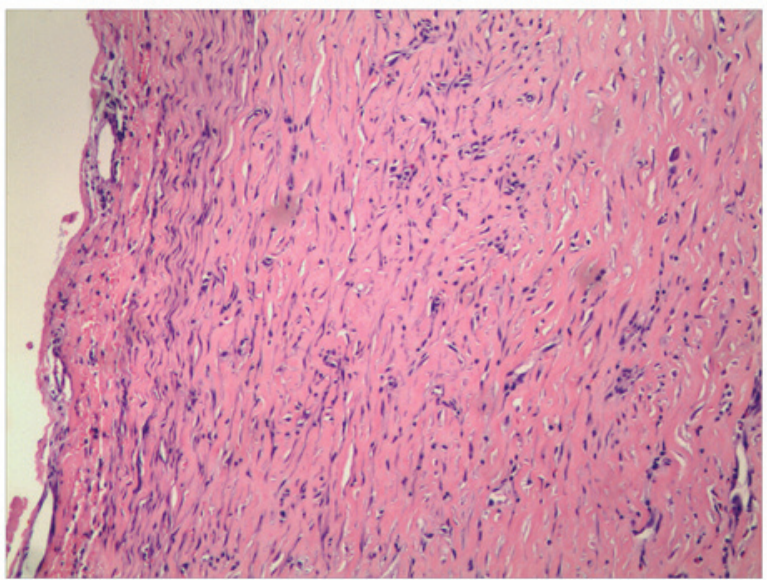

Figure 4. Routine pathology demonstrated that the capsule comprised fibrous tissue without epithelial lining (hematoxylin and eosin stain; magnification, $\mathrm{x} 100)$.

Under such circumstances, a frozen section examination is mandatory to differentiate benign from malignant lesions, and the definitive diagnosis should be based on histological examination (26). As such, suspected BCTs should be treated with surgical resection when feasible.

As for biloma, without timely intervention, the collection may progress to an infected abscess, and become fatal. Management of individual patients depends on the etiologies, symptoms, tumor-associated factors and the medical condition of the patient. Typically small and asymptomatic bilomas can 
be monitored periodically whereas, in cases with large-sized and infected lesions, or where the patient is medically unfit for surgery, percutaneous drainage is the appropriate approach, and may suffice for the evacuation of bilomas. Adjunct therapies, including percutaneous transhepatic biliary drainage (PTBD) and an endoscopic technique, are mandatory to facilitate healing of the defect in persistent cases, and these yield satisfactory results $(15,27,28)$. As a general rule, endoscopic intervention is responsive for perforations located distally to the confluence of the left and right hepatic duct, whereas PTBD is responsive for perforations located proximally to the confluence. Surgical treatment may be warranted in cases refractory to minimally invasive management, or in dealing with the etiology.

There are few reports on biloma formation due to GBP, but all cases have featured radiological discontinuity of the gallbladder wall $(13,15,16)$. Tiny extravasation of unconcentrated bile from the gallbladder and relatively large subphrenic space should have been responsible for the insidious symptoms, normal laboratory tests and undetected perforation site in our patient, and preoperative diagnosis represented a challenge. To the best of our knowledge, this is the first report of a giant and asymptomatic subphrenic biloma formation after calculus cholecystitis without visualized defects and mimicking a biliary cystic tumor.

In conclusion, GBP is subdivided into three categories, whereas the formation of biloma is rare, and should be classified as type IV (13). A pinhole-sized perforation with extravasation of unconcentrated bile from the gallbladder may result in insidious clinical presentation and an undetected leak site. Preoperative differentiation of bilomas with intracystic septations and mural nodules from BCTs is difficult, and the definitive diagnosis should be based on histological analysis of the resected specimen. Laparotomy with a frozen section examination may be the optimal approach for discriminating benign from malignant lesions in cases of patients who are fit for surgery.

\section{Acknowledgements}

The present study was supported by the China Postdoctoral Science Foundation (no. 2015M581837).

\section{References}

1. Kannan U, Parshad R and Regmi SK: An unusual presentation of biloma five years following cholecystectomy: A case report. Cases J 2: 8048, 2009.

2. Göbel T, Kubitz R, Blondin D and Häussinger D: Intrahepatic type II gall bladder perforation by a gall stone in a CAPD patient Eur J Med Res 16: 213-216, 2011.

3. Derici H, Kara C, Bozdag AD, Nazli O, Tansug T and Akca E: Diagnosis and treatment of gallbladder perforation. World J Gastroenterol 12: 7832-7836, 2006.

4. Ausania F, Guzman Suarez S, Alvarez Garcia H, Senra del, Rio P and Casal Nuñez E: Gallbladder perforation: Morbidity, mortality and preoperative risk prediction. Surg Endosc 29: 955-960, 2015

5. Seyal AR, Parekh K, Gonzalez-Guindalini FD, Nikolaidis P, Miller FH and Yaghmai V: Cross-sectional imaging of perforated gallbladder. Abdom Imaging 39: 853-874, 2014.

6. Gunasekaran G, Naik D, Gupta A, Bhandari V, Kuppusamy M, Kumar G and Chishi NS: Gallbladder perforation: A single center experience of 32 cases. Korean J Hepatobiliary Pancreat Surg 19: 6-10, 2015.
7. Ohtake T, Kimura M, Yoshii S, Ikegaya N, Takayanagi S, Hishida A and Kaneko E: Biloma during steroid therapy for minimal change nephrotic syndrome. Intern Med 32: 543-546, 1993.

8. Niemeier OW: Acute free perforation of the gall-bladder. Ann Surg 99: 922-924, 1934

9. Date RS, Thrumurthy SG, Whiteside S, Umer MA, Pursnani KG, Ward JB and Mughal MM: Gallbladder perforation: Case series and systematic review. Int J Surg 10: 63-68, 2012.

10. Gould L and Patel A: Ultrasound detection of extrahepatic encapsulated bile: 'Biloma'. AJR Am J Roentgenol 132: 1014-1015, 1979.

11. Kuligowska E, Schlesinger A, Miller KB, Lee VW and Grosso D: Bilomas: A new approach to the diagnosis and treatment. Gastrointest Radio 8: 237-243, 1983.

12. Copelan A, Bahoura L, Tardy F, Kirsch M, Sokhandon F and Kapoor B: Etiology, diagnosis, and management of bilomas: A current update. Tech Vasc Interv Radiol 18: 236-243, 2015.

13. Kalfadis S, Ioannidis O, Botsios D and Lazaridis C: Subcapsular liver biloma due to gallbladder perforation after acute cholecystitis. J Dig Dis 12: 412-414, 2011.

14. Akhtar MA, Bandyopadhyay D, Montgomery HD and Mahomed A: Spontaneous idiopathic subcapsular biloma. J Hepatobiliary Pancreat Surg 14: 579-581, 2007.

15. Tsai MC, Chen TH, Chang MH, Chen TY and Lin CC: Gallbladder perforation with formation of hepatic subcapsular biloma, treated with endoscopic nasobiliary drainage. Endoscopy 42: (Suppl 2) E206-E207, 2010.

16. Ferrusquía-Acosta JA, Álvarez-Navascués $\mathrm{C}$ and Rodríguez-García M: Giant biloma as a result of a blunt abdominal trauma: A case report. Rev Esp Enferm Dig 107: 768-769, 2015.

17. Lee CM, Stewart L and Way LW: Postcholecystectomy abdominal bile collections. Arch Surg 135: 538-544, 2000.

18. Ziessman HA: Hepatobiliary scintigraphy in 2014. J Nucl Med 55: 967-975, 2014.

19. Mbarushimana S, Morris-Stiff G and Hassn A: CT diagnosis of an iatrogenic bile duct injury. BMJ Case Rep 2014: bcr2014204918, 2014 .

20. Ishii K, Matsuo K, Seki H, Yasui N, Sakata M, Shimada A and Matsumoto H: Retroperitoneal Biloma due to Spontaneous Perforation of the Left Hepatic Duct. Am J Case Rep 17: 264-267, 2016.

21. Doussot A, Gluskin J, Groot-Koerkamp B, Allen PJ, De Matteo RP, Shia J, Kingham TP, Jarnagin WR, Gerst SR and D'Angelica MI: The accuracy of pre-operative imaging in the management of hepatic cysts. HPB (Oxford) 17: 889-895, 2015.

22. LeeCW,TsaiHI,Lin YS, WuTH, YuMC and Chen MF: Intrahepatic biliary mucinous cystic neoplasms: Clinicoradiological characteristics and surgical results. BMC Gastroenterol 15: 67, 2015.

23. Soares KC, Arnaoutakis DJ, Kamel I, Anders R, Adams RB, Bauer TW and Pawlik TM: Cystic neoplasms of the liver: Biliary cystadenoma and cystadenocarcinoma. J Am Coll Surg 218: 119-128, 2014.

24. Kakisaka T, Kamiyama T, Yokoo H, Nakanishi K, Wakayama K, Tsuruga Y, Kamachi H, Mitsuhashi T and Taketomi A: An intraductal papillary neoplasm of the bile duct mimicking a hemorrhagic hepatic cyst: A case report. World J Surg Oncol 11: $111,2013$.

25. Kishida N, Shinoda M, Masugi Y, Itano O, Fujii-Nishimura Y, Ueno A, Kitago M, Hibi T, Abe Y, Yagi H, et al: Cystic tumor of the liver without ovarian-like stroma or bile duct communication: Two case reports and a review of the literature. World J Surg Oncol 12: 229, 2014.

26. Arnaoutakis DJ, Kim Y, Pulitano C, Zaydfudim V, Squires MH, Kooby D, Groeschl R, Alexandrescu S, Bauer TW, Bloomston M, et al: Management of biliary cystic tumors: A multi-institutional analysis of a rare liver tumor. Ann Surg 261: 361-367, 2015.

27. Attasaranya S, Netinasunton N, Jongboonyanuparp T, Sottisuporn J, Witeerungrot T, Pirathvisuth T and Ovartlarnporn B: The Spectrum of Endoscopic Ultrasound Intervention in Biliary Diseases: A Single Center's Experience in 31 Cases. Gastroenterol Res Pract 2012: 680753, 2012.

28. de Jong EA, Moelker A, Leertouwer T, Spronk S, Van Dijk M and van Eijck $\mathrm{CH}$ : Percutaneous transhepatic biliary drainage in patients with postsurgical bile leakage and nondilated intrahepatic bile ducts. Dig Surg 30: 444-450, 2013. 